\title{
Visceral Kaposi's Sarcoma Related to Human Herpesvirus-8 in Liver Transplant Recipient: Case Report and Literature Review
}

\author{
H. Benhammane, ${ }^{1}$ G. Mentha, ${ }^{2}$ E. Tschanz, ${ }^{3}$ O. El Mesbahi, ${ }^{1}$ and P. Y. Dietrich ${ }^{4}$ \\ ${ }^{1}$ Department of Medical Oncology, Hassan II University Hospital, Fez, Morocco \\ ${ }^{2}$ Department of Transplantation, University Hospitals of Geneva, 1205 Geneva, Switzerland \\ ${ }^{3}$ Department of Pathology, University Hospitals of Geneva, 1205 Geneva, Switzerland \\ ${ }^{4}$ Department of Medical Oncology, University Hospitals of Geneva, 1205 Geneva, Switzerland \\ Correspondence should be addressed to H. Benhammane, hafida.benhammane@gmail.com
}

Received 2 September 2012; Accepted 9 December 2012

Academic Editors: S. Aksoy and J. Keller

Copyright (๑) $2012 \mathrm{H}$. Benhammane et al. This is an open access article distributed under the Creative Commons Attribution License, which permits unrestricted use, distribution, and reproduction in any medium, provided the original work is properly cited.

\begin{abstract}
Background. Kaposi's sarcoma (KS) in transplant recipients is about 400 to 500 times rate in the general population. It is strongly associated to Human herpesvirus-8 (HHV-8) infection which has been found in 95\% of KS lesions. The optimal approach to managing posttransplantation KS is to reduce or discontinue immunosuppressive therapy but this strategy carries a risk of the acute rejection of the graft. Recently, the use of an mTOR inhibitor has added new opportunities for KS treatment and prevention. Case Report. We report a case of 24 years-old Turkish woman with visceral HHV-8-associated Kaposi's sarcoma after orthotopic liver transplantation. Conclusion. Posttransplantation KS is considered an experimental model of virus induced tumor suggesting the usefulness of HHV-8 screening in transplant recipient and donor. Therapeutic approaches are complex and require a multidisciplinary team.
\end{abstract}

\section{Introduction}

Organ transplantation is associated with an increased risk of malignancies; Kaposi's sarcoma (KS) accounts for 5,7\% of these malignancies [1]; it is more common in liver transplant recipients than in other solid organ transplantation; in a large Italian cohort on 417 liver transplant recipient, KS accounts for $14 \%$ of postliver transplant malignancies [2, $3]$. This neoplasia is characterized by the predominance of skin and mucosal lesions; visceral extension is seen in only $10 \%$ of cases $[2,4,5]$. Human herpes virus- 8 (HHV-8) is considered an essential, although not sufficient, etiologic agent for the development of KS; in the setting of organ transplantation the pathogenesis of this oncogenic virus remains not clearly understood [6]. The reduction or cessation of immunosuppressive therapy should be the first therapeutic maneuver of post-transplantation $\mathrm{KS}[1,7]$; recently, some reports demonstrate that the use of an mTOR inhibitor may be associated with a complete regression $[8,9]$. Chemotherapy is an option for no responding patients. The role of antiviral therapy against $\mathrm{HHV}-8$ is still controversial
[6]. Herein, we report a case of visceral HHV-8-associated KS in 24 years-old woman after orthotopic liver transplantation (OLT) in order to underline the features and outcomes of this malignancy and to review the role of HHV-8.

\section{Case Report}

A 24 years-old Turkish woman underwent OLT in November 2008 for cirrhosis related to Von Gierke disease. Her immunosuppressive regimen consisted of tacrolimus and mycophenolate mofetil. Three years later, she was readmitted because of a cholestasis due to the stenosis of the biliobiliary anastomosis. Endoscopic Retrograde Cholangiopancreatography with placement of biliary stent was performed, but the procedure was complicated by pancreatitis and duodenal ulcer; therefore, a biliary repair surgery was decided. A computed tomography performed preoperatively allowed to fortuitous detection of several pulmonary nodules and multiple intraperitoneal lymph nodes (Figure 1). The patient underwent laparotomy which demonstrated the presence of 


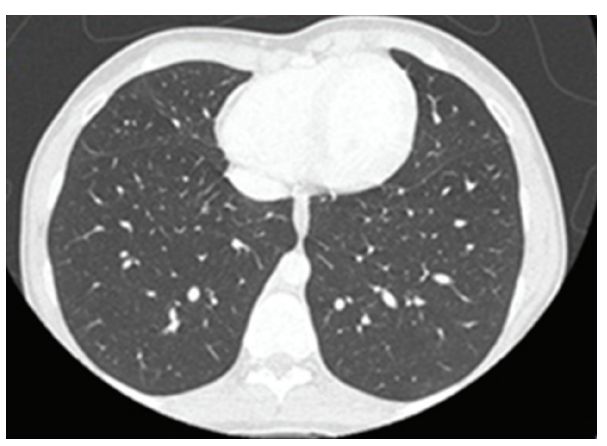

(a)

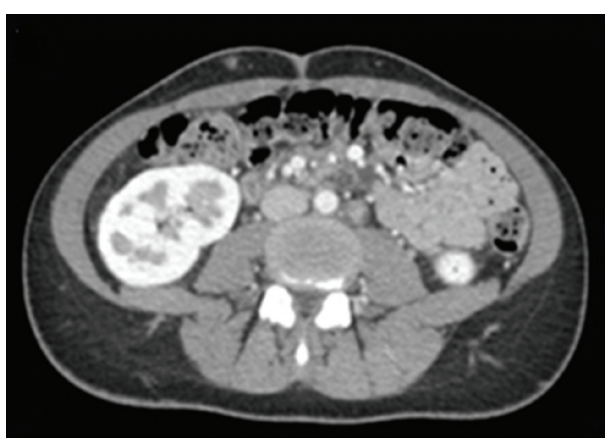

(b)

FIGURE 1: Computed tomography showing multiple pulmonary nodules (a) and retroperitoneal lymph nodes (b).

multiple lymph nodes of variable size, associated with a $3 \mathrm{~cm}$ sized lesion of small bowel; principal bile duct was dilated secondary to a biliary nevrome. Segmental resection of small bowel with lymphadenectomy was performed; biliary stenosis was treated by hepaticojejunal anastomosis on Roux-en-Y. Histological analysis confirmed the diagnosis of kaposi's sarcoma of small bowel with lymph nodes extension (Figure 2); tumoral cells showed strong positivity in the nuclei for HHV-8, CD34 and CD31 (Figure 3). Antibody testing for HHV-8 was reactive on the serum bank collected on day 5 of OLT excluding a donor related infection. The serum HHV-8 viral load was 989 copies/Ml. other viral serology (HIV, hepatitis B and C virus, Epstein-Barr virus and cytomegalovirus) was negative. The immunosuppressive regimen was initially reduced, by stopping mycophenolate mofetil and halving the oral tacrolimus daily dose from 5 to $2 \mathrm{mg}$ during 1 month then it was converted to everolimus at a daily dose of $2 \mathrm{mg}$. Currently, the patient remains well without sign of progression disease 9 months after surgery and with a good tolerance of treatment.

\section{Discussion}

Kaposi's sarcoma (KS) is about 400-500 times more common in transplant recipients than in general population [7], with an overall incidence of $0,4-6 \%$ in the United States and Western Europe $[1,10]$. KS following organ transplantation and immunosuppressive therapy was first published in 1969 [11]. As reported in the Cincinnati Transplant Tumor Registry (CTTR), It accounts for 5 to $7 \%$ of all malignancies in transplant recipients when nonmelanoma skin cancer and in situ carcinoma of the uterine cervix are excluded [1]. $70 \%$ of posttransplant KS are diagnosed in the first 2 years after receiving transplantation and the most cases occur in individuals of Mediterranean, Jewish, Arabic, black, and Greek descent [6].

The incidence of KS is greater in liver transplant recipients $(1,24 \%)$ than in heart $(0,41 \%)$ or kidney $(0,45 \%)$ transplantation $[3,4,6]$ due to different types of immunosuppressive therapy. Clinical features are characterized by a predominance of cutaneous and mucosal lesions as multiple vascular nodules; an extension to viscera or lymph nodes

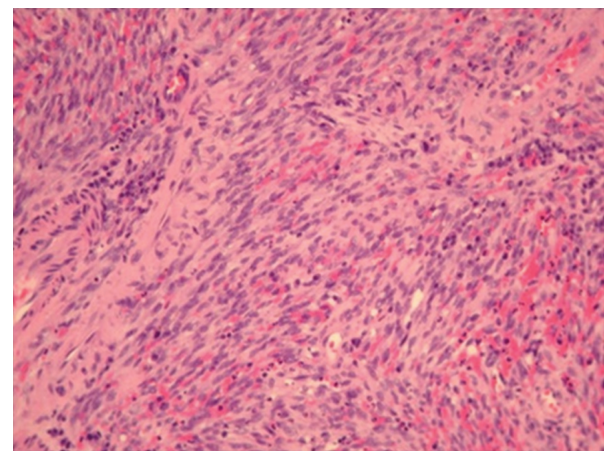

Figure 2: Microscopic findings of small bowel specimen showing atypical spindle cells with extravasated erythrocytes and capillaries $($ HES $\times 100)$.

is observed in only $10 \%$ of cases which $50 \%$ occur in liver transplant recipient $[2,4,5]$. Visceral disease has a substantial mortality ranging from $30-60 \%$ [1].

Although the pathogenesis of KS in transplant patients is poorly understood, a strong correlation with immunosuppressive status and the reactivation of HHV-8 was demonstrated. In 1994, HHV-8 has been isolated in all four types of KS (classic, endemic, organ transplant-associated, and AIDS-related KS) [6]. Over 95\% of KS lesions, regardless of their clinical type, have been found to be infected with HHV-8. this oncogenic virus is an herpesviridae family strongly associated to KS and other conditions especially Castleman's disease and primary effusion lymphoma. In the endemic area for HHV-8, graft recipients who develop KS are generally seropositive before transplantation as is the case of our patient, but a transmission from donors to recipients has been documented [12]. The following explanations have been suggested: viral reactivation due to immunosuppressive therapy and chronic immunologic reaction between altered lymphoid cells and normal lymphocytes; in fact, HHV8 infects both lymphatic and blood vascular endothelial cells and seems to target immunosuppressor tumor proteins (retinoblastoma and p53) causing the production of lymphangiogenic growth factors, (bFGF, Scatter factor, IL6, etc), endothelial cell proliferation, and tumorigenesis [12, 13]. Therefore post-transplantation KS represents actually an 


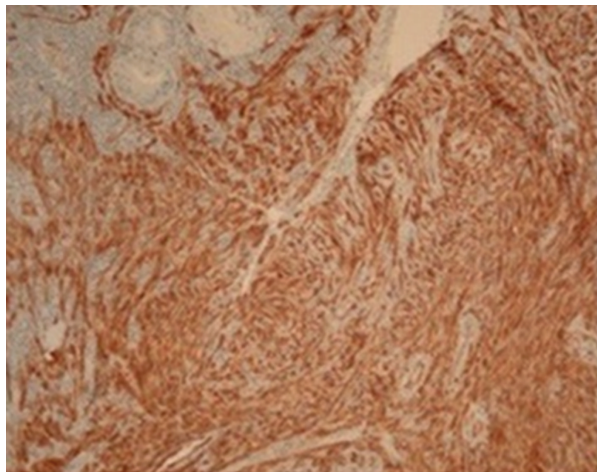

(a)

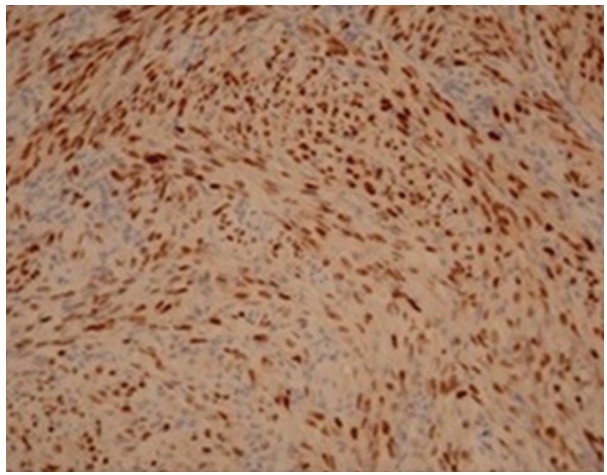

(b)

FIGURE 3: Immunohistochemical analysis showing the positivity for CD31 and CD34 (a) and the strong immunoreactivity for HHV-8 in the nuclei (b).

experimental model of virus induced tumor as a consequence of prolonged immunosuppression.

Although, infection with HHV-8 is essential but not sufficient for the development of KS in transplant recipient, several cofactors have been identified including extent and duration of immunosuppression, reduced levels of neutralizing antibodies, poor cytotoxic T-cell response to HHV-8, and concomitant viral infection (HIV, hepatitis B virus, EpsteinBarr virus, cytomegalovirus, and herpes simplex virus) $[12,13]$; additionally, when post-transplantation is more common in some ethnic background, several reports suggest a genetic predisposition related to some HLA phenotype [12]; however, because of the absence of specific studies on HLA phenotype in organ transplant recipient and donor, this hypothesis remains controversial.

No consensus on the optimal treatment for posttransplantation KS is available; therapeutic choice is often made based upon the experience and medical discipline; many treatment modalities have been used: surgical excision, radiation therapy, intralesional injection of chemotherapeutic agents, reduction of immunosuppressive therapy and systemic chemotherapy. Because the disease is a consequence of prolonged immunosuppression, the reduction or cessation of immunosuppressive therapy may be the most effective and reasonable approach $[1,6,12]$, in the CTTR, a complete remission was observed in $42 \%$ of patients after various treatment; $38 \%$ of these remissions followed the reduction or cessation of immunosuppressive therapy; nonvisceral disease was associated to a higher remission rate than visceral disease (53\% versus 27\%) [1]. Similar results were reported by other authors [7, 12]. However, the management of immunosuppression is difficult particularly in KS following liver or heart transplantation [12] and requires a balance between the risk of death from neoplasia and the risk of graft rejection due to under immunosuppression [7].

Recently, some data suggest that switching to immunosuppressive therapy using a (Sirolimus or Everolimus) can induce the regression of iatrogenic KS through antiangiogenic activity related to impaired VEGF production and the limited proliferative response of endothelial cells to VEGF stimulation [8,9]; additionally, L. A. Nichols showed a probable role of mTOR inhibitor in the prevention of $\mathrm{KS}$ in immunosuppressed patient by blocking $\mathrm{HHV}-8$ production via the regulation of replication and transcription activator expression [14]. Given the complete regression reported in several published cases, we think that mTOR inhibitor should be included in the treatment of post transplantation KS. When the reduction or cessation of immunosuppressive therapy failed, conventional chemotherapy can be used. Pegylated liposomal doxorubicin is considered to be the first line therapy of choice leading to $70 \%$ of complete or major response approximately [15]. The other cytotoxic agents that seem to be effective in KS are vinblastine, bleomycin, taxane, etoposide, and gemcitabine. There are a few data on the role of antiviral therapy against $\mathrm{HHV}-8$, but in combination with other treatments, they may allow to a satisfactory response as noted in some available observational reports [16].

\section{Conclusion}

The significant increase in the incidence of KS in transplant recipients shows the role of the immune system in the control of HHV-8 infection which is considered the principal, but not sufficient, etiologic agent for this neoplasia; there is evidence that screening transplant recipients and blood donors for HHV-8 infection may be beneficial. Further, studies are needed to identify immunologic, infectious and genetic cofactors possibly involved. The reduction of immunosuppression is a useful therapeutic approach of post-transplantation $\mathrm{KS}$; the introduction of an mTOR inhibitor may permit the remission of the sarcoma; however, the total regression of the lesions should not be the goal because of the risk of graft rejection. In fact, therapeutic approaches are complex and require a skilled, experienced, multidisciplinary team.

\section{Acknowledgments}

All authors have made significant contributions by making diagnosis, treatment, and intellectual input in the case and writing the paper. The authors declare that they have no competing interests. 


\section{References}

[1] I. Penn, "Sarcomas in organ allograft recipients," Transplantation, vol. 60, no. 12, pp. 1485-1491, 1995.

[2] D. Farge, "Kaposi's sarcoma in organ transplant recipients," European Journal of Medicine, vol. 2, no. 6, pp. 339-343, 1993.

[3] U. Baccarani, P. Piselli, D. Serraino et al., "Comparison of de novo tumours after liver transplantation with incidence rates from Italian cancer registries," Digestive and Liver Disease, vol. 42, no. 1, pp. 55-60, 2010.

[4] M. R. Moosa, "Kaposi's sarcoma in kidney transplant recipients: a 23-year experience," Quarterly Journal of Mathematics, vol. 98, no. 3, pp. 205-214, 2005.

[5] P. Aseni, M. Vertemati, E. Minola et al., "Kaposi's sarcoma in liver transplant recipients: morphological and clinical description," Liver Transplantation, vol. 7, no. 9, pp. 816-823, 2001.

[6] R. A. Schwartz, G. Micali, M. R. Nasca, and L. Scuderi, "Kaposi sarcoma: a continuing conundrum," Journal of the American Academy of Dermatology, vol. 59, no. 2, pp. 179-206, 2008.

[7] S. Duman, H. Töz, G. Aşçi et al., "Successful treatment of posttransplant Kaposi's sarcoma by reduction of immunosuppression," Nephrology Dialysis Transplantation, vol. 17, no. 5, pp. 892-896, 2002.

[8] G. Stallone, B. Infante, G. Grandaliano, F. P. Schena, and L. Gesualdo, "Kaposi's sarcoma and mTOR: a crossroad between viral infection neoangiogenesis and immunosuppression," Transplant International, vol. 21, no. 9, pp. 825-832, 2008.

[9] C. Lebbé, S. Euvrard, B. Barrou et al., "Sirolimus conversion for patients with posttransplant Kaposi's sarcoma," American Journal of Transplantation, vol. 6, no. 9, pp. 2164-2168, 2006.

[10] P. Piselli, G. Busnach, F. Citterio et al., "Risk of Kaposi sarcoma after solid-organ transplantation: multicenter study in 4767 recipients in Italy, 1970-2006," Transplantation Proceedings, vol. 41, no. 4, pp. 1227-1230, 2009.

[11] J. H. Siegel, R. Janis, J. C. Alper, H. Schutte, L. Robbins, and M. D. Blaufox, "Disseminated visceral Kaposi's sarcoma. Appearance after human renal homograft operation," Journal of the American Medical Association, vol. 207, no. 8, pp. 1493 1496, 1969.

[12] I. Penn, "Kaposi's sarcoma in transplant recipients," Transplantation, vol. 64, no. 5, pp. 669-673, 1997.

[13] Y. K. Hong, K. Foreman, J. W. Shin et al., "Lymphatic reprogramming of blood vascular endothelium by Kaposi sarcoma-associated herpesvirus," Nature Genetics, vol. 36, no. 7, pp. 683-685, 2004.

[14] L. A. Nichols, L. A. Adang, and D. H. Kedes, "Rapamycin blocks production of KSHV/HHV8: insights into the antitumor activity of an immunosuppressant drug," PLoS ONE, vol. 6, no. 1, Article ID e14535, 2011.

[15] R. di Trolio, G. di Lorenzo, M. Delfino, and S. de Placido, "Role of pegylated lyposomal doxorubicin (PLD) in systemic Kaposi's sarcoma: a systematic review," International Journal of Immunopathology and Pharmacology, vol. 19, no. 2, pp. 253263, 2006.

[16] G. Verucchi, L. Calza, F. Trevisani et al., "Human herpesvirus8-related Kaposi's sarcoma after liver transplantation successfully treated with cidofovir and liposomal daunorubicin," Transplant Infectious Disease, vol. 7, no. 1, pp. 34-37, 2005. 


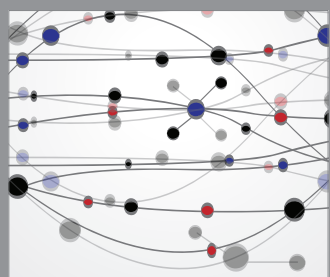

The Scientific World Journal
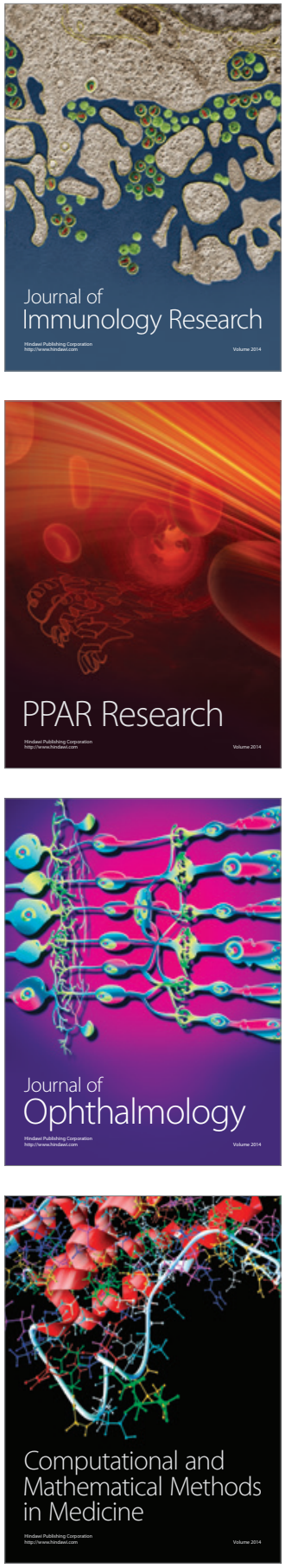

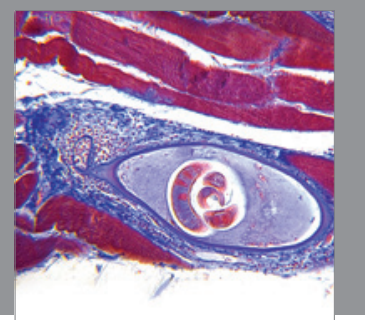

Gastroenterology

Research and Practice
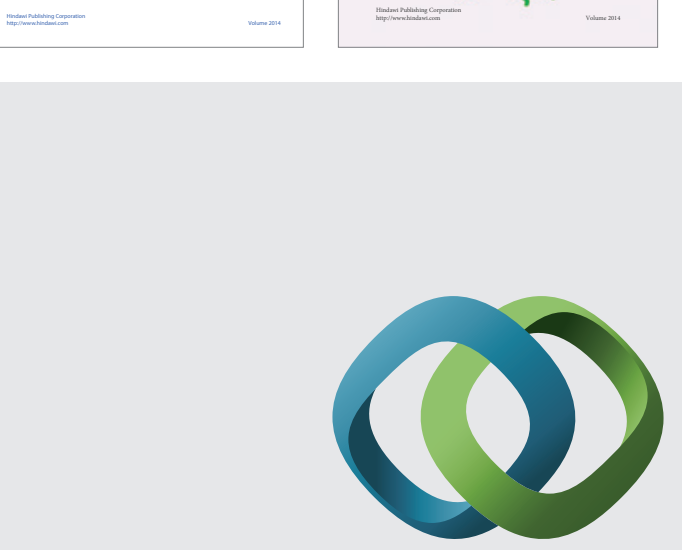

\section{Hindawi}

Submit your manuscripts at

http://www.hindawi.com
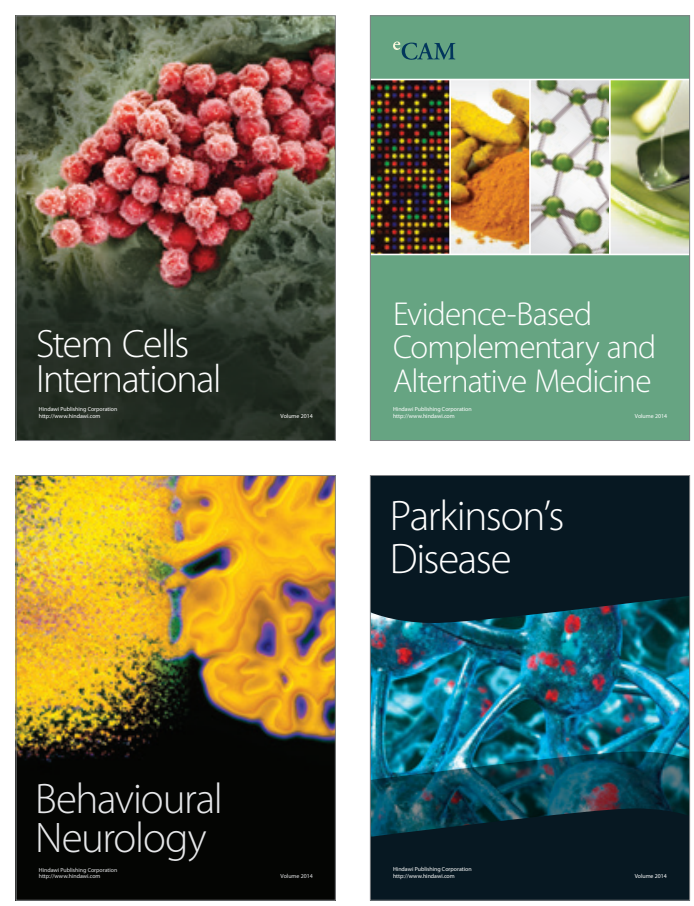

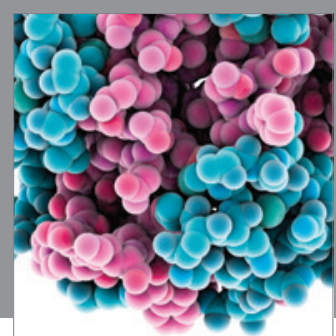

Journal of
Diabetes Research

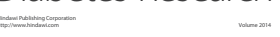

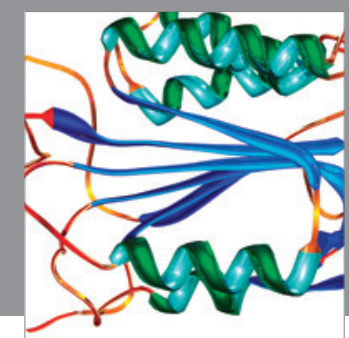

Disease Markers
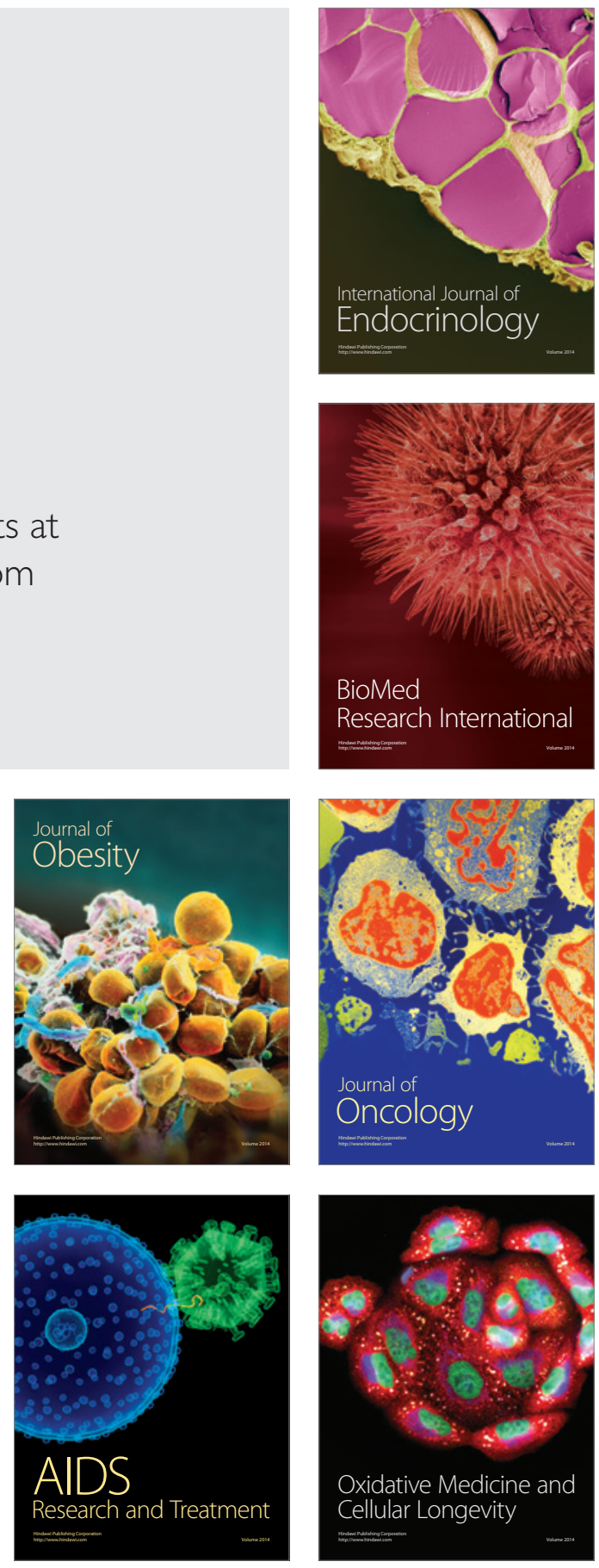